\title{
Medicion de la calidad del aire en la cuenca del lago de patzcuaro mediante el uso de un red de monitoreo perimetral
}

\author{
Measurement of air quality in the patzcuaro lake basin using a perimeter \\ monitoring network
}

DOI: $10.46932 /$ sfjdv2n5-130

Received in: Oct 1st, 2021

Accepted in: Dec 30th, 2021

\section{Tania Paulina Pulido Varela}

Estudiante del Instituto Tecnológico Superior de Pátzcuaro y miembro de la red ambiental interinstitucional en colaboración del proyecto CONACYT 316204.

Mariquita Linda \#63, Pátzcuaro, Michoacán, México.

E-mail: taniapaulinapulidovarela@gmail.com

\section{Mariela De La Cruz Almanza}

Estudiante del Instituto Tecnológico Superior de Pátzcuaro y miembro de la red ambiental interinstitucional en colaboración del proyecto CONACYT 316204.

Ignición Allende \# 27, Santa María Huiramangaro, Michoacán, México.

E-mail: marydlc9819@gmail.com

\section{Félix Aldair Cázarez Yépez}

Estudiante del Instituto Tecnológico Superior de Pátzcuaro y miembro de la red ambiental interinstitucional en colaboración del proyecto CONACYT 316204.

Fray Alonso de Larrea \#64, Pátzcuaro, Michoacán, México.

E-mail: aldaircazarez007@gmail.com

\section{Marcial Reyes Cázarez}

Doctor en Género y Derecho.

Tecnológico Nacional de México campus Pátzcuaro.

Investigador Independiente del Estado de Michoacán REIM_RECM81HMGE02

Av. Tecnológico $\mathrm{N}^{\circ} 1$. Tzurumutaro, Pátzcuaro, Michoacán, México.

E-mail: mreyes@itspa.edu.mx / marcial.rc@patzcuaro.tecnm.mx

\section{RESUMEN}

Se pretende llevar a cabo una red de monitoreo en la cuenca del lago de Pátzcuaro con la finalidad de medir la calidad del aire tomando en cuenta los contaminantes atmosféricos presentes en suelo y agua, a causa de que en el Estado de Michoacán no se da un correcto monitoreo en cuestiones de calidad. Mediante el uso de sensores para medir los siguientes parámetros. Aire: CO2, O3, CH4, Material Particulado.

Suelo: Temperatura, Humedad, Conductividad eléctrica. Agua: Temperatura, Sólidos Suspendidos Totales, $\mathrm{pH}$, Conductividad eléctrica. Los resultados que se pretenden obtener son tomar mediciones no solo atmosféricas sino también en áreas como el agua y el suelo, de manera que sea posible un monitoreo completo de los contaminantes, desarrollar un dispositivo autónomo en la obtención de datos atmosféricos, para llevar un control estadístico de los mismos y lograr una estación amable con la naturaleza y que no sea intimidante con la fauna, dándole un aspecto de flora nativa.

Palabras Clave: Monitoreo, Medición, Calidad, Recursos Naturales, Red de Sensores. 


\begin{abstract}
It is intended to carry out a monitoring network in the Patzcuaro Lake basin in order to measure the air quality taking into account the atmospheric pollutants present in soil and water, because in the State of Michoacan there is no proper monitoring in quality issues. By using sensors to measure the following parameters. Air: CO2, O3, CH4, Particulate Matter. Soil: Temperature, Humidity, Electrical conductivity. Water: Temperature, Total Suspended Solids, $\mathrm{pH}$, Electrical conductivity. The intended results are to take measurements not only in atmospheric but also in areas such as water and soil, so that a complete monitoring of pollutants is possible, to develop an autonomous device to obtain atmospheric data, to keep a statistical control of them and to achieve a friendly station with nature and that is not intimidating to wildlife, giving it an aspect of native flora.
\end{abstract}

Keywords: Monitoring, Measurement, Quality, Natural Resources, Sensor Network.

\title{
1 INTRODUCCIÓN
}

Las estaciones de monitoreo permiten llevar un registro diario de las emisiones que son generadas por las distintas fuentes emisoras, por lo cual se tiene una estimación adecuada de la contaminación producida dependiendo del área y numero de fuentes emisoras de ese punto especifico.

Es necesario contar con datos estadísticos de la actividad diaria, que generan una gran cantidad de sustancias que modifican la composición natural del aire, suelo y agua. El deterioro de la calidad de los elementos principales por la presencia de sustancias contaminantes tiene un efecto negativo en la salud humana y el medio ambiente. La modificación de nuestro hábitat toma un papel importante. Diversos estudios han demostrado que existe una relación entre el incremento en la concentración de los contaminantes del aire, agua y suelo provocan un aumento de enfermedades respiratorias, cardiovasculares, entre otras.

Esta información es fundamental para establecer medidas de control de la contaminación y políticas para proteger la salud de la población y del medio ambiente, principalmente en lugares donde no se encuentran estaciones de monitoreo atmosférico.

En la región de la Riviera del Lago de Pátzcuaro hace falta la determinación de estos factores de emisión, ya que estas estaciones se hacen más presentes en lugares más grandes como las capitales de los estados, por lo tanto no se tiene la estadística de las emisiones en lugares de vital importancia como lo son los Lagos emblemáticos de nuestro estado, en este caso para el desarrollo del proyecto se busca implementar una estación de monitoreo que no solo tome en cuenta las emisiones al aire, sino también las que llegan al agua y al suelo, esto debido a que en las estaciones de monitoreo que se usan en las grandes ciudades no toman en cuenta que algunos de estos contaminantes descienden a estas dos partes por lo que el dato estadístico está incompleto y no permite tener un dato real de la contaminación de la zona. 
La estación de monitoreo contribuirá al monitoreo local, donde se incluyan las herramientas necesarias para que este instrumento sea accesible y disponible a la mayoría de usuarios. Detectando las principales amenazas ambientales percibidas por las comunidades locales y las variables o parámetros que se deberían controlar o monitorear para establecer medidas de prevención y control.

La calidad del agua de ríos, presas o lagos, del aire o suelo pueden ser descrita a partir de la combinación de más de dos variables. Variables como los principales parámetros que describen las condiciones físicas y químicas del agua. Estos parámetros proporcionarán la información mínima necesaria para realizar una evaluación de la calidad del agua, lo que a su vez permitirá generar información valiosa y poder almacenar en una base de datos.

\section{ANTECEDENTES}

En las últimas dos décadas la problemática ambiental dentro del estado de Michoacán se ha visto reflejada en distintas partes del mismo, solo por mencionar en la cuenca del lago de Pátzcuaro en cual vemos un gran problema de contaminación hídrica, respecto a monitoreo del mismo se tiene poca información toda enfocada en estudios hídricos realizados para proyectos u obras dentro del estado de Michoacán, por lo cual es necesario implementar un sistema de monitoreo. Con el objetivo de tener una mayor representatividad en la red de monitoreo, en el año 2012 surgió una necesidad de crear una red de monitoreo en otros lugares de la república como lo son Estado de México, la cual fuera capaz de realizar la determinación de la calidad del agua con las frecuencias y parámetros adecuados en los principales cuerpos de agua. Lo anterior, permitiría evaluar los efectos del cambio climático, atender sitios de interés nacional e internacional, tales como zonas turísticas, humedales (áreas naturales protegidas, entre otros), e implementar sitios de monitoreo en las cuencas y acuíferos que sean necesarios con el objeto de proteger las fuentes de abastecimiento de un mayor porcentaje de la población, así como el hábitat de la flora y fauna acuática. Esta idea surgió desde el año 1996 frente las limitaciones de recursos materiales y humanos y los problemas de cobertura de los sitios de monitoreo de los principales cuerpos de agua del país, se planteó la tarea de rediseñar la Red Nacional de Monitoreo de la Calidad del Agua, con el objetivo principal de generar información representativa y confiable de la calidad del agua de una manera costoefectiva más eficiente.

Dentro del parámetro de suelo podemos encontrar que dentro de México se encuentran en desarrollo proyectos subsidiados por el Consejo Nacional de Ciencia y Tecnología (CONACYT) en donde se establecen condiciones preliminares para el establecimiento de una red de monitoreo de contaminantes en suelo (PROYECTO CONACYT-PRONASES 316204 [detección de herbicidas selectivos]) por lo cual se toma en cuenta antecedentes destacados de otros países como lo es en Europa en donde se desarrolló el proyecto SOILCAM («Contaminación del suelo: caracterización avanzada integrada y monitorización 
a intervalos», financiado por la UE, se propuso mejorar esta situación. Este proyecto se centró en diseñar la combinación óptima de métodos invasivos y no invasivos para monitorear contaminantes degradables en diferentes suelos.

SOILCAM se concentró en dos sitios de campo en Europa que enfrentan problemas de contaminación: Trecate en el norte de Italia (petróleo ligero) y Gardermoen en el sur de Noruega (productos químicos de deshielo). Las mediciones geofísicas a intervalos se combinaron con métodos de recopilación de datos de un solo punto.

Los enfoques geofísicos dan una interpretación de las propiedades, pero en una escala relativa. Sin embargo, los datos puntuales proporcionan una medición absoluta de las propiedades químicas y físicas. La combinación de estos métodos permitió al proyecto SOILCAM determinar los diferentes niveles de contaminantes y su propagación en los diferentes entornos de los dos sitios de campo.

La experiencia y el conocimiento obtenidos a través del proyecto SOILCAM pueden proporcionar una contribución útil para ayudar a la implementación de la política europea de protección del suelo. Además, dado que tanto las aguas subterráneas como las superficiales pueden contaminarse por lugares contaminados, los resultados del proyecto son aún más relevantes para la política del agua y la gestión de recursos hídricos de Europa.

Finalmente, dentro del parámetro atmosférico podemos encontrar antecedentes del SINAICA que es una serie de programas informáticos que permiten recabar, transmitir y publicar la información de calidad del aire que se genera en las estaciones de monitoreo ubicadas en las diversas entidades federativas que disponen de la infraestructura adecuada para tal tipo de medición. La información proviene de Sistemas de Monitoreo de Calidad del Aire, SMCA, que son manejados por diferentes órdenes de gobierno, estatal y municipal. Las mediciones de calidad del aire de los SMCA se pueden consultar en dos diferentes secciones:

- Datos crudos de calidad del aire y de variables meteorológicas en tiempo real.

- Indicadores de calidad del aire. Esta sección incluye además la visualización y descarga de los datos históricos validados.

\section{OBJETIVOS}

La estación de monitoreo nos permitirá determinar los niveles de calidad del aire, del suelo, así como del agua y las condiciones meteorológicas que favorecen el transporte y la dispersión de los contaminantes en una región dada. Dentro de este proyecto lo importante es desarrollar un dispositivo autónomo en la obtención de datos atmosféricos, para llevar un control estadístico de los mismos. De manera que sea posible lograr una estación amable con la naturaleza y que no sea intimidante con la fauna, dándole un aspecto de flora nativa. Además de ayudar a las autoridades e instituciones con datos relevantes 
para la aplicación de medidas y políticas en el beneficio de la biodiversidad en la región. Para así tomar en cuenta mediciones no solo atmosféricas sino también en áreas como el agua y el suelo, de manera que se obtenga un monitoreo completo de los contaminantes. Por lo cual será necesario crear una base de datos en la cual se lleve un control de monitoreo de la contaminación y políticas para proteger la salud de la población y del medio ambiente, principalmente en lugares donde no se encuentran estaciones de monitoreo atmosférico.

materiales: Los materiales que se utilizaran para la elaboración de nuestro proyecto son los siguientes:

- Termómetro - Higrómetro - Sensor Humedad

- Sensor De Temperatura Y Humedad (Dht11 C/jumpers Arduino)

- Termopar Tipo K Sensor Temperatura (y Modulo Max6675)

- Sensor De Humedad Del Suelo, Temperatura y Conducta Eléctrica

- Arduino Uno R3

- Modulo Sensor Calidad De Aire (Detector Mq135 Arduino)

- Sensor Óptico De Polvo y material particulado Arduino

- Btmeter Lcd Digital - Detector De La Calidad Del Aire

- Sensor De Turbiedad Y Calidad Del Agua

- Sensor De PH Con Modulo Arduino

- Medidor Tds-ec Conductividad Eléctrica, Ppm, Solidos Disueltos

- Modelos en 3D para camuflar los sensores y no invadan el hábitat natural

○ Modelo de Roca (Fig. 1).

○ Modelo de Nenúfar (Fig. 2 y 3).

○ Modelo de Estróbilo de Pino (Fig. 4).
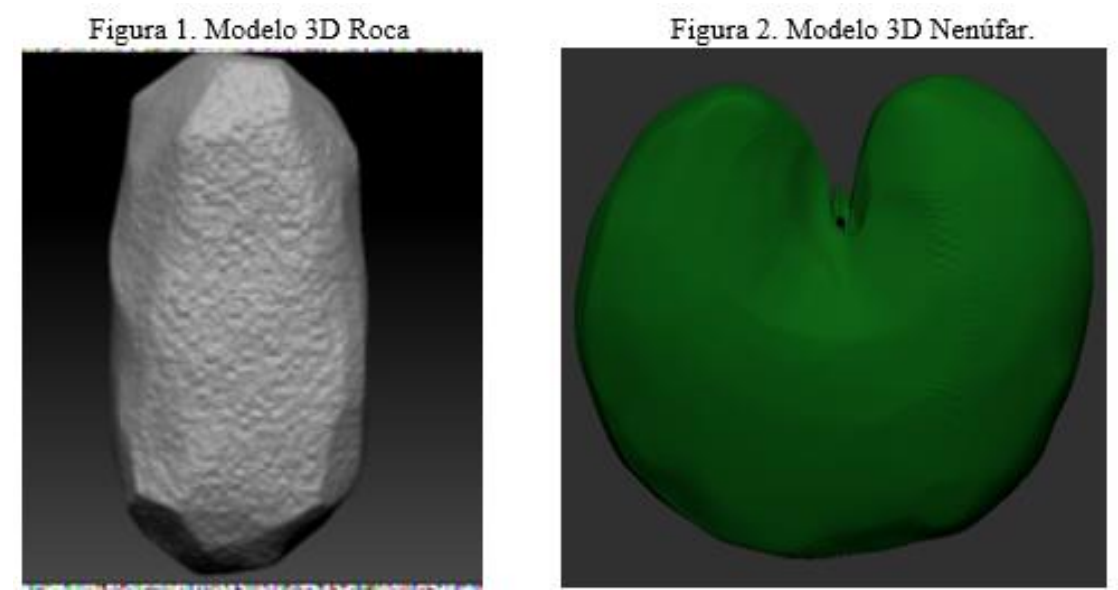

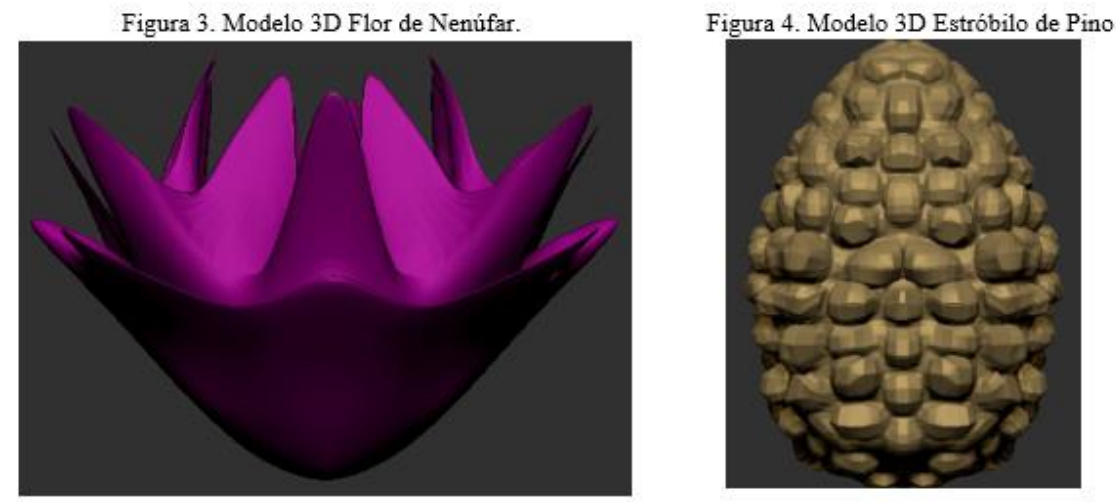

\section{MÉTODO}

Se colocarán las estaciones con sus respectivos sensores en los tres sitios que se monitorearán, el agua, suelo y aire, ayudados de los modelos para camuflarlos en las áreas y no perturben a las formas de vida ahí presentes, los adecuaremos para que estos se vean de manera natural y discreta. Después se programará una aplicación móvil que nos permitirán conocer las condiciones de emisiones al momento gracias a que cada sensor arroja sus datos de manera remota, los cuales se alojaran en un servidor que los distribuirá a la aplicación al momento, para poder tener los datos de la frecuencia en que sean necesarios, ya sea cada hora, cada día, semana, etc. Permitiéndonos tener un dato estadístico preciso que nos permita saber de manera adecuada la calidad del aire, agua y suelo, para tomar las medidas preventivas necesarias

\section{RESULTADOS}

En los cuerpos de agua analizados se ha un incremento de $\mathrm{pH}$ arriba de lo normal, el agua debe tener de 5.8 y 6.8 y se tiene un pH por arriba de 6.8, principalmente por la elevada concentración de coliformes fecales, se ha visto un alza en la temperatura de los cuerpos de agua teniendo una temperatura de aproximadamente $19.7^{\circ} \mathrm{C}$. Los contaminantes atmosféricos como monóxido y dióxido de carbono se han incrementado significativamente en la atmósfera, ocasionando el incremento en la temperatura con respecto al tiempo, $\mathrm{n}$ las ciudades de Morelia, Zamora y Maravatío se ha visto un incremento en los últimos 3 años de $0.5{ }^{\circ} \mathrm{C}$ y tenemos una tendencia creciente. La disminución en la cubierta vegetal y volúmenes de árboles ha ocasionado en menor infiltración de agua de lluvia al subsuelo y pérdida de diversidad de especies. La humedad en el suelo se ha determinado al $60 \%$ en las zonas de cultivo; donde existe vegetación de pino y encino tenemos una humedad del 84\%; en la zona centro del estado donde se encuentran especies exóticas invasivas como la casuarina y el eucalipto, el análisis obtenido promedió tan solo el 34\% de humedad en el suelo. Al tener menor humedad en el suelo, el pH se incrementa teniendo consecuencias en la productividad o restauraciones de esos espacios, la T del suelo ha incrementado $2{ }^{\circ} \mathrm{C}$ en las zonas boscosas y $1.6^{\circ} \mathrm{C}$ en zonas de cultivo, la diferencia se da porque en la zona de cultivo todo 
el año se les induce la humedad, al tener mayor humedad en el suelo la conductividad eléctrica será mayor humedad mayor conductividad eléctrica entre las plantas. Afectando el $\mathrm{pH}$ del suelo, afectando las reacciones químicas que determinan si los nutrientes estarán o no disponibles (solubles o insolubles) para su absorción.

\section{CONCLUSIONES}

Es necesario diseñar e implementar un sistema efectivo de monitoreo ambiental en el estado que nos permita generar una base de datos con la cual contar para poder tomar las decisiones necesarias, diseñar acciones de prevención y remediación en salvaguarda de los recursos naturales y el medio que nos rodea. Dichas acciones deben realizarse en conjunto por la legislación y la ciudadanía.

Es necesario establecer una red de calidad del aire, de calidad de suelo y de calidad hidrológica en donde establezcamos criterios respecto a los volúmenes de vegetación arbórea y arbustiva nativa en donde se establezca el monitoreo, en donde el equilibrio ecológico y la protección al ambiente sean los factores primordiales para detonar acciones de restauración o conservación según convengan.

Existen alteraciones por tecnologías utilizadas para la producción de frutos para la agroexportación que generan alteraciones al ciclo hídrico y a la propia condición climática de las microrregiones en donde se implementan, tal es el caso de los sistemas de protección anti granífugos (Reyes Cázarez, M., Pulido Varela, T., \& Cázarez Yépez, F. (2019). SISTEMAS DE PROTECCIÓN ANTIGRANÍFUGOS EN MÉXICO Y SUS EFECTOS EN LA SALUD DE LOS SERES VIVOS Y LAS ALTERACIONES AMBIENTALES (CAÑONES ANTIGRANIZO). Medicina: Impactos Científicos E Sociais E Orientação A Problemas Nas Diversas Áreas De Saúde 2.) y de las avionetas antilluvia, así como el uso excesivo de agroquímicos.

El tema del agua en México es un tema de seguridad nacional y tenemos que visualizar que ya existen conflictos en diferentes zonas geográficas por la escasez o mala calidad de este recurso. Asimismo, la Organización Mundial de la Salud determina que la falta de agua y/o el consumo de agua contaminada van en constante incremento, lo que provoca la transmisión de enfermedades como el cólera, otras diarreas, hepatitis A, disintiera, fiebre tifoidea y la poliomielitis y una vez más coincide que quienes tienen limitaciones para acceder al agua potable o apta para consumo humano son aquellos que se encuentran con menores posibilidades económicas.

La contaminación atmosférica ha provocado en la población mexicana que el 15\% padezca problemas de tipo respiratorio, lo que a corto plazo comenzara a generar problemas en las grandes ciudades, es importante mencionar también que otro de los factores asociados a este tipo de problemas son las condiciones de vida de las familias y una vez más aquellas personas con menores ingresos y que viven en situación de pobreza extrema son las más perjudicadas. 


\section{REFERENCIAS}

ALONSO, E., \& MARTÍNEZ, W., \& RUBIO, J. C., \& VELASCO, F., \& CHÁVEZ, H. L., \& ÁVALOS, M., \& LARA, C., \& CERVANTES, E. (2007). Calidad del Aire en Cuatro Ciudades de Michoacán, México: Su Efecto sobre Materiales de Construcción. Revista de la Construcción, 6(2),66-74. [fecha de Consulta 9 de octubre de 2020]. ISSN: 0717-7925. Disponible en: https://www.redalyc.org/articulo.oa?id=1276/127619405007

Cordis.europa.eu. 2012. CORDIS | European Commission. [online] Available at: <https://cordis.europa.eu/article/id/90059-an-integrated-approach-to-soil-pollution-monitoring> [fecha de Consulta 8 de octubre de 2020].

Dgeiawf.semarnat.gob.mx. 2019. SEMARNAT. [online] Available at: <http://dgeiawf.semarnat.gob.mx:8080/ibi_apps/WFServlet?IBIF_ex=D3_R_AGUA05_03\&IBIC_user =dgeia_mce\&IBIC_pass=dgeia_mce> [fecha de Consulta 8 de octubre de 2020].

Granados-Ramírez, Rebeca, \& Longar Blanco, María del Pilar (2008). Variabilidad pluvial, agricultura y marginación en el estado de Michoacán. Análisis Económico, XXIII (54),283-303. [fecha de Consulta 9 de octubre de 2020]. ISSN: 0185-3937. Disponible en: https://www.redalyc.org/articulo.oa?id=413/41311483014

Gob.Mx. Retrieved December 16, 2021, from https://icti.michoacan.gob.mx/wpcontent/uploads/2019/06/Revista-No.-20.-AWEB.pdf

Gopar-Merino, Luis Fernando, \& Velázquez, Alejandro (2016). Componentes del paisaje como predictores de cubiertas de vegetación: estudio de caso del estado de Michoacán, México. Investigaciones Geográficas (Mx), (90),75-88. [fecha de Consulta 9 de octubre de 2020]. ISSN: 0188-4611. Disponible en: https://www.redalyc.org/articulo.oa?id=569/56946869007

Madrigal, Xavier, \& Novelo, Alejandro, \& Chacón, Arturo (2004). Flora y vegetación acuáticas del lago de Zirahuén, Michoacán, México. Acta Botánica Mexicana, (68),1 - 38. [fecha de Consulta 9 de octubre de 2020]. ISSN: 0187-7151. Disponible en: https://www.redalyc.org/articulo.oa?id=574/5740680

Sinaica.inecc.gob.mx. 2020. INSTITUTO NACIONAL DE ECOLOGÍA Y CAMBIO CLIMÁTICO. [online] Available at: <https://sinaica.inecc.gob.mx/> [fecha de Consulta 8 de octubre de 2020].

Reyes Cázarez, M., Granados Melchor J. A., Tovar Aguilar R. (2020). MANEJO SUSTENTABLE DE LA VEGETACIÓN ARBOREA Y RECONVERSIÓN DEL PAISAJE EN PATZCUARO, MICHOACÁN, MÉXICO. ENTORNOS DESDE EL INTERIOR: Estudios Sobre El Paisaje. 